\title{
Isolated cotton-wool spot during pregnancy: A case report
}

\author{
Praveena K Gupta* and Giovanni Taibbi \\ Department of Ophthalmology and Visual Sciences, The University of Texas Medical Branch at Galveston, Galveston, TX, USA
}

\begin{abstract}
Introduction: The presence of cotton-wool spots warrants a full systemic workup. We present a case of an isolated cotton-wool spot in a pregnant woman with visual changes after a recent flu-like illness.

Case Report: A 25 year-old Middle Eastern female at the $20^{\text {th }}$ gestational age with a past medical history of Lyme disease, presented with a $24-$ hour history of a sudden onset of a dark, horizontal line in the center of her right eye vision. She experienced a flu-like illness while on a recent trip to Puerto Rico. Dilated fundus exam revealed an isolated cotton-wool spot along the superior temporal arcade of the right eye, producing focal Retinal Nerve Fiber Layer thickness. Buried optic disc drusen were detected by ocular ultrasound bilaterally. Inflammatory/infectious blood workup, including antibody against Zika virus, was negative except for a mild increase of ANA titer (1:40) in the absence of rheumatologic disease. The patient was managed conservatively and no treatment was initiated. At 4-month follow-up, there was a nearly complete resolution of the cotton-wool spot in the absence of any visual symptoms. The patient had a full-term uncomplicated vaginal delivery.

Discussion: The presence of cotton-wool spots, alone or in combination with other clinical findings, warrants further investigation. Although most cotton-wool spots resolve without long-term visual sequelae and could be managed with close observation only, they have been associated with life- or sight-threatening conditions. Therefore, any underlying causes should be identified and treated appropriately. Given the absence of a pre-specified workup for cotton-wool spots, the extent and type of workup should be dictated by the appropriate clinical context.
\end{abstract}

\section{Introduction}

Cotton-wool spots result from interruption of the axoplasmic flow of the retinal ganglion cells, leading to axoplasmic debris accumulation [1]. They are most commonly seen in patients with diabetes mellitus or systemic hypertension. However, cotton-wool spots have also been associated with cardiac valve disease, hematological/neoplastic (eg, anemia, hyperviscosity syndromes, multiple myeloma, leukemia) [2,3] inflammatory (eg, Systemic Lupus Erythematous [SLE], giant cell arteritis), or infectious (eg, HIV) [4] disorders. On the other hand, they have also been reported as isolated findings in otherwise healthy individuals. Because cotton-wool spots may be seen in life- or sightthreatening conditions, their presence should prompt further clinical investigation. We present a case of an isolated cotton-wool spot in a pregnant woman returning from a trip to Puerto Rico.

\section{Case report}

A 25-year old Middle Eastern pregnant female at the $20^{\text {th }}$ week of gestation and a past medical history of Lyme disease (negative antibody titer) presented to the clinic with a chief complaint of acute, stationary dark horizontal line through the central visual field of her right eye for the previous 24 hours. The line appeared as white upon closure of the eyes. She was a soft contact lens wearer and used topical cyclosporine for dry eyes. The rest of her past ocular history was unremarkable. The patient had returned from a recent trip to Puerto Rico, where she developed a flu-like illness. At the time of initial presentation, the patient was still complaining of mild fatigue, perhaps from her recent trip. The remainder of the review of systems was negative and blood pressure was reported as normal.

On examination, distance visual acuity was $20 / 20$ in each eye with contact lenses. Ocular motility was full with no deviations, diplopia or nystagmus. Confrontation visual field was significant for a horizontal central scotoma in the right eye; it was full in the left eye. The pupils were round and reactive to the light; no anisocoria or relative afferent pupillary defect was detected. Intraocular pressure was $10 \mathrm{mmHg}$ in each eye by applanation tonometry. Slit-lamp examination was unremarkable in either eye. Dilated fundus exam revealed marked right optic disc elevation with sharp margins and pink neuro-retinal rim, and an isolated cotton-wool spot adjacent to the supero-temporal vascular arcade (Figure 1). The left optic disc was notable for a mild elevation and blurriness of the nasal disc margin, with pink neuro-retinal rim throughout (Figure 1). The macula was unremarkable in either eye.

The patient was referred to our Retina clinic the next day for further evaluation. Spectral-Domain OCT at the level of the right cotton-wool spot revealed increased retinal nerve fiber layer thickness (Figure 2). Given the funduscopic appearance of the optic discs, further assessment with orbital ultrasound was performed, which revealed a few buried optic disc drusen bilaterally. Blood workup included the following: complete blood count, blood glucose, antinuclear antibody (ANA) titer, HIV and syphilis serology, Bartonella Hensalae IgG-IgM, CMV IgG-IgM, Zika virus IgM titer (given the patient's trip to Puerto Rico during the Zika virus epidemic). Lyme disease antibody test was not ordered due to documented negative titers. The patient maintained full visual acuity in each eye, and no treatment was initiated. The

*Correspondence to: Praveena Gupta, OD, PhD, FAAO, Associate Professor, Department of Ophthalmology and Visual Sciences, The University of Texas Medical Branch at Galveston, 700 University Blvd, Galveston, TX, 77550, USA, E-mail:prgupta@utmb.edu

Key words: cotton-wool spot, Lyme disease, optic disc drusen, pregnancy, Zika virus

Received: May 18 2020; Accepted: June 04, 2020; Published: June 09, 2020 
blood workup was negative, except for a mild elevation of the ANA titer (1:40). Therefore, the patient was referred to Rheumatology: she did not meet the diagnostic criteria for SLE, and the presence of other autoimmune/rheumatologic disease was ruled out. There was no indication for initiation of medical treatment, and the patient continued to be managed conservatively by close observation only.
At 1-month follow-up, the patient noted fading of the central, horizontal dark line in the right eye. At 4-month follow-up, there was a nearly complete resolution of the patient's baseline visual symptoms and regression of the right eye cotton-wool spot, as confirmed by dilated fundus exam and Spectral Domain OCT imaging (Figure 3). Ultimately, the patient underwent uncomplicated full-term vaginal delivery.
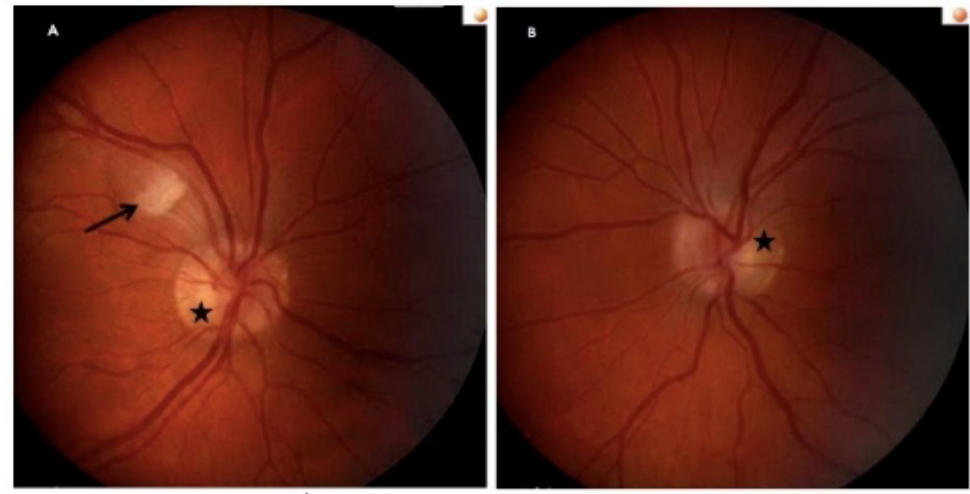

Figure 1. Color fundus photographs of the right eye (A) and the left eye (B). In the right eye, a fluffy cotton-wool spot adjacent to the superior temporal vascular arcade is seen (arrow). In each eye, buried optic disc drusen (asterisks) provide a typical pseudo-papilledema appearance.
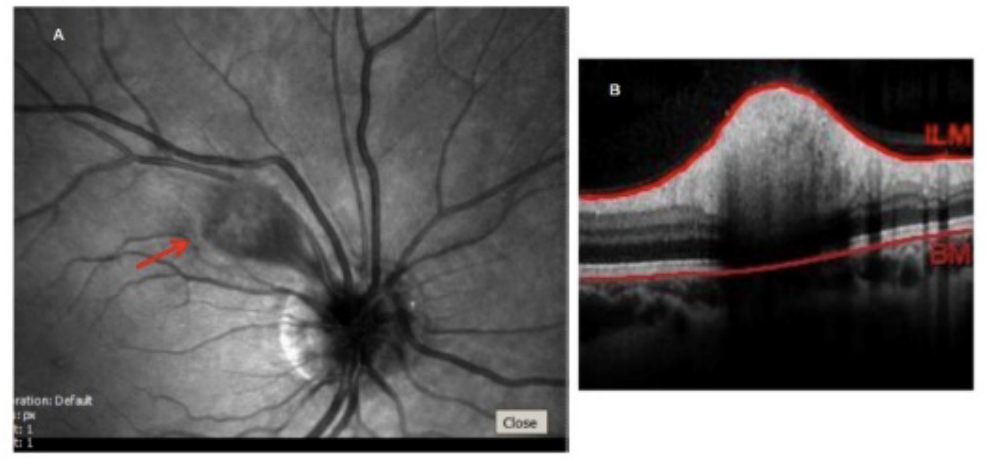

Figure 2. Right eye cotton-wool spot appearance on infrared imaging (A, arrow), and corresponding cross-sectional Spectral-Domain OCT scan across the cotton-wool spot (B) showing increased retinal nerve fiber thickness (total retinal thickness: $521 \mu \mathrm{m}$ ).
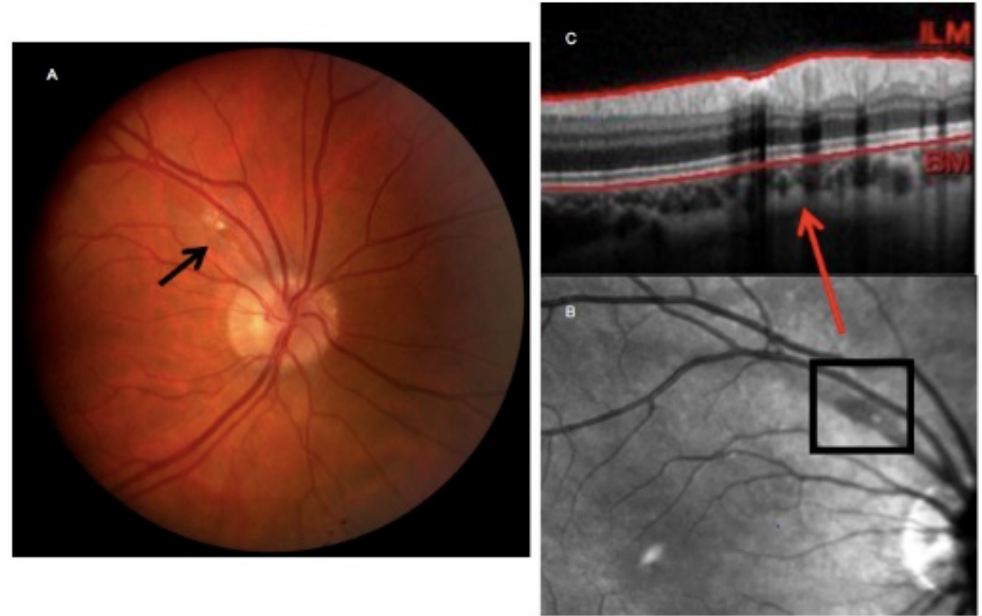

Figure 3. (A) Color fundus photograph of the right eye showing near-complete resolution of the cotton-wool spot at 4-month follow-up (see Figure 1 for comparison). The corresponding infrared image and Spectral-Domain OCT scan are shown in (B) and (C), respectively (see Figure 2 for comparison). At 4-month follow-up, the total retinal thickness at the level of the cotton-wool spot decreased to $328 \mu \mathrm{m}$. 


\section{Discussion}

We presented a case of a pregnant patient with a history of Lyme disease returning from a recent trip to Puerto Rico, who presented with a dark, horizontal line in her central vision associated with an isolated cotton-wool in the right eye. The patient was managed conservatively by observation only, with resolution of both her visual complaint and the cotton-wool spot at 4-month follow-up.

As previously reported, the presence of an isolated cotton-wool spot in an otherwise healthy individual should not be overlooked [1]. A thorough systemic workup may uncover life-threatening conditions, such as multiple myeloma [2], or lead to the diagnosis of idiopathic cotton-wool spot [5].

We ordered a comprehensive blood workup, which was negative except for a mild increase in the ANA titer (1:40). Isolated cottonwool spots have been reported in SLE patients [6]; however, there was no clinical evidence of SLE or other immunological/rheumatologic condition. Although rarely, cotton-wool spots have been observed during pregnancy [7]. Possible underlying mechanisms include focal ischemic retinal events resulting from the hypercoagulable state of pregnancy, along with pregnancy-related hormonal changes [7].

To our knowledge, cotton-wool spot have not been reported in association with ocular Zika. However, cotton-wool spots have been found in retinitis secondary to Dengue virus, a member of the Flaviviridae family comprising the Zika virus [8]. Therefore, it was important to rule out ocular Zika in our patient given her recent stay in an area of Zika epidemic. Given the absence of a pre-specified workup for cotton-wool spots, the extent and type of diagnostic workup should be dictated by the appropriate clinical context.

Patients with cotton-wool spots may or may not present with visual symptoms [9], depending on the underlying ocular or systemic disorder. However, the location of the cotton-wool spot may also provide insight on the expected degree of visual complaints. For example, since the axons of the retinal nerve fibers converge at the level of the optic nerve head, any cotton-wool spots closer to the optic disc margin may indicate blockage of the axoplasmic flow to a higher number of retinal nerve fibers [1], possibly leading to a greater degree of visual disturbance when compared to cotton-wool spots located away from the optic disc or in a more peripheral retinal location. In our patient, the visual disturbance in her right eye appeared to be correlated with the size of the cotton-wool spot. In agreement with the literature [10], the cotton-wool spot resolved without sequelae over the course of a 4 -month period.

In conclusion, the detection of cotton-wool spots, alone or in combination with other clinical findings, warrants further investigation. Although most cotton-wool spots resolve without long-term sequelae and could be managed with close observation only, any underlying causes should be identified and treated appropriately.

\section{Acknowledgments}

None

\section{Funding information}

None

\section{Competing interests}

The authors declare that they have no competing interests

\section{References}

1. Brown GC, Brown MM, Hiller T, Fischer D, Benson WE, Magargal LE (1985) Cottonwool spots. Retina 5: 206-214. [Crossref]

2. Shami MJ, Uy RN (1996) Isolated cotton-wool spots in a 67-year-old woman. Surv Ophthalmol 40: 413-415.

3. Abu el-Asrar AM, al-Momen AK, Kangave D, Harakati MS (1995) Prognostic importance of retinopathy in acute leukemia. Doc Ophthalmol 91: 273-281. [Crossref]

4. Geier SA, Klauss V, Goebel FD (1994) Ocular microangiopathic syndrome in patients with acquired immunodeficiency syndrome and its relationship to alterations in cell adhesion and in blood flow. Ger J Ophthalmol 3: 414-421. [Crossref]

5. Ioannides A, Georgakarakos ND, Elaroud I, Andreou P (2011) Isolated cotton-wool spots of unknown etiology: management and sequential spectral domain optical coherence tomography documentation. Clin Ophthalmol 5: 1431-1433. [Crossref]

6. Gold DH, Morris DA, Henkind P (1972) Ocular findings in systemic lupus erythematosus. Br J Ophthalmol 56: 800-804.

7. Remky A, Arend O (2000) Single isolated cotton-wool spots. Ophthalmologica 214: 143-148.

8. de Andrade GC, Ventura CV, Mello Filho PA, Maia M, Vianello S, Rodrigues EB (2017) Arboviruses and the eye. Int J Retina Vitreous 3: 4. [Crossref]

9. Bek T, Lund-Andersen H (1991) Cotton-wool spots and retinal light sensitivity in diabetic retinopathy. Br J Ophthalmol 75: 13-17. [Crossref]

10. Kohner EM, Dollery CT, Bulpitt CJ (1969) Cotton-wool spots in diabetic retinopathy. Diabetes 18: 691-704.

Copyright: (C2020 Gupta PK. This is an open-access article distributed under the terms of the Creative Commons Attribution License, which permits unrestricted use, distribution, and reproduction in any medium, provided the original author and source are credited. 